\title{
Distance measure and its application
}

\author{
Thangaraj Beaula ${ }^{1 *}$ and Selvakumari ${ }^{2}$
}

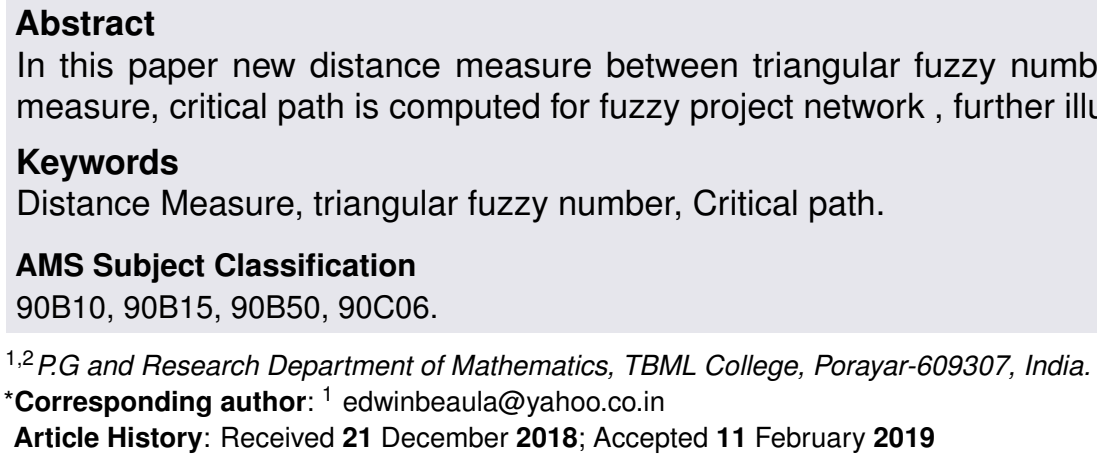

\section{Contents}

1 Introduction ...........................66

2 Preliminaries ..........................66

3 New distance between two fuzzy numbers........67

4 Application in critical path method ............67

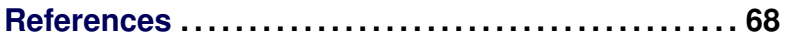

\section{Introduction}

The theory of fuzzy sets was first introduced by Zadeh in 1965 which opened new gates to the field of applied mathematics. The concept of fuzzy numbers was also introduced by Zadeh which is widely spread in the area of research in fuzzy mathematics. The distance between the fuzzy numbers plays an important role in fields like remote sensing, data mining, pattern recognition. Usually the fuzzy distance is computed in terms of crisp distance. Voxman[6] was the first to coin fuzzy distance between fuzzy numbers.

In this paper a new distance measure between triangular fuzzy numbers is established and using the new distance measure critical path is computed for fuzzy project network. A new algorithm is developed to obtain the critical path, illustrated by a numerical example.

\section{Preliminaries}

Definition 2.1. A fuzzy set on a set $X$ is a function $\mu: X \rightarrow$ $[0,1]$. The support of $\mu$ is the closure of the set $\{x \in X / \mu(x)>$ $0\}$ usually denoted by supp $\mu$.
Definition 2.2. A fuzzy number is a fuzzy set $\mu: \mathbb{R} \rightarrow[0,1]$ on $\mathbb{R}$ satisfying

1. $\mu$ is upper semi-continuous.

2. supp $\mu$ is a closed and bounded interval.

3. If supp $\mu=[a, b]$ then there exist $c, d, a \leq c \leq d \leq b$ such that $\mu$ is increasing on the interval[a,c], equal to 1 , on the interval $[c, d]$ and decreasing on the interval[d,b].

Definition 2.3. Let $F$ denote the family of all fuzzy numbers. If $\mu \in F$ then for each $\alpha, 0<\alpha \leq 1$, the $\alpha$-cut of $\mu$ is defined by $\mu_{\alpha}=\{x \in X / \mu(x) \geq \alpha\}$

The $\alpha$-cut representation of $\mu$ is the pair of functions $(L(\alpha), R(\alpha))$ defined by

$L(\alpha)= \begin{cases}\inf \left\{x / x \in \mu_{\alpha}\right\}, & \text { if } \alpha>0 \\ \inf \{x / x \in \operatorname{supp} \mu\}, & \text { if } \alpha=0 .\end{cases}$

and

$R(\alpha)= \begin{cases}\sup \left\{x / x \in \mu_{\alpha}\right\}, & \text { if } \alpha>0 \\ \sup \{x / x \in \operatorname{supp} \mu\}, & \text { if } \alpha=0 .\end{cases}$

Definition 2.4. A fuzzy number $\tilde{A}=(a, b, c)$ is said to be a triangular fuzzy number if it is membership function is given by

$$
\mu_{\tilde{A}}(x)= \begin{cases}\frac{x-a}{b-a}, & a \leq x \leq b \\ 1, & x=b \\ \frac{c-x}{c-b}, & b \leq x \leq c \\ 0, & \text { otherwise. }\end{cases}
$$

The $\alpha$-cut representation of a triangular fuzzy number $\tilde{A}$ is $A_{\alpha}=\left[A^{L}(\alpha), A^{R}(\alpha)\right]$

$$
A_{\alpha}=[a+\alpha(b-a), c-\alpha(c-b)]
$$




\subsection{Fuzzy Distance (Voxman)}

Describe the fuzzy distance measure by Voxman[6] as a function $\triangle: F \times F \rightarrow F$

$$
\begin{aligned}
\triangle(\mu, v)(z) & =\operatorname{Supmin}\{\mu(x), v(y) \\
|x-y| & =z
\end{aligned}
$$

For each pair of fuzzy numbers $\mu$ and $\mathrm{v}$, let $\Delta \mu v$ denote the fuzzy number $\Delta(\mu, v)$. If the $\alpha$-cut representations of $\mu$ and $\mathrm{v}$ are $\left(A_{1}^{L}(\alpha), A_{1}^{R}(\alpha)\right)$ and $\left(A_{2}^{L}(\alpha), A_{2}^{R}(\alpha)\right)$ respectively then the $\alpha$ - cut representation of $\Delta_{\mu v},(L(\alpha), R(\alpha))$ is given by

$L(\alpha)=\max \left\{A_{2}^{L}(\alpha)-A_{1}^{R}(\alpha), 0\right\}$ if $1 / 2\left(A_{1}^{L}(1)+A_{1}^{R}(1)\right) \leq 1 / 2\left(A_{2}^{L}+A_{2}^{R}(1)\right)$

$=\max \left\{A_{1}^{L}(\alpha)-A_{2}^{R}(\alpha), 0\right\}$ if $1 / 2\left(A_{2}^{L}(1)+A_{2}^{R}(1)\right) \leq 1 / 2\left(A_{1}^{L}+A_{1}^{R}(1)\right)$ and

$$
R(\alpha)=\max \left\{A_{1}^{R}(\alpha)-A_{2}^{L}(\alpha), A_{2}^{R}(\alpha)-A_{1}^{L}(L(\alpha))\right\}
$$

\section{New distance between two fuzzy numbers}

Definition 3.1. Let $A_{1}$ and $A_{2}$ be two arbitrary fuzzy numbers with $\alpha$-cut representation $\alpha_{A_{1}}=\left[A_{1}^{L}(\alpha)-A_{1}^{R}(\alpha)\right]$ and $\alpha_{A_{2}}=$ $\left[A_{2}^{L}(\alpha)-A_{2}^{R}(\alpha)\right]$ respectively. The new distance between $A_{1}$ and $A_{2}$ is defined as $d\left(\alpha_{A_{1}}, \alpha_{A_{2}}\right)=\left[\left(\text { a.c }-A_{1}^{L}(\alpha)\right)^{2}+\left(A_{1}^{R}-\right.\right.$ a.c $\left.)^{2}-\left[\left(\text { a.c }-A_{2}^{L}(\alpha)\right)^{2}+\left(A_{2}^{R}-\text { a.c }\right)^{2}\right]\right]^{1 / 2}$

and

$D\left(A_{1}, A_{2}\right)=\int_{0}^{1 / 2}(1-\alpha) d\left(\alpha_{A_{1}}, \alpha_{A_{2}}\right) d_{\alpha}+\int_{1 / 2}^{1} \alpha d\left(\alpha_{A_{1}}, \alpha_{A_{2}}\right) d_{\alpha}$

where a.c $=\frac{L(\alpha)+R(\alpha)}{2}$

Property 3.2. $d^{2}\left(\alpha_{\left(A_{1}+A_{2}\right)}, \tilde{0}\right)<d^{2}\left(\alpha_{A_{1}}, \tilde{0}\right)+d^{2}\left(\alpha_{A_{2}}, \tilde{0}\right)$ for every $\alpha_{A_{1}}, \alpha_{A_{2}} \in F$

Proof.

$$
\begin{aligned}
& d^{2}\left(\alpha_{\left(A_{1}+A_{2}\right)}, \tilde{0}\right)=\left(a . c-\left(A_{1}^{L}+A_{2}^{L}\right)\right)^{2}+\left(\left(A_{2}^{R}+A_{2}^{R}\right)-a . c\right)^{2} \\
& =(a . c)^{2}+\left(A_{1}^{L}+A_{2}^{L}\right)^{2}-2 a . c\left(A_{1}^{L}+A_{2}^{L}\right) \\
& +\left(A_{1}^{R}+A_{2}^{R}\right)^{2}+(a . c)^{2}-2 a \cdot c\left(A_{1}^{R}+A_{2}^{R}\right) \\
& =(a . c)^{2}+\left(A_{1}^{L}\right)^{2}+\left(A_{2}^{L}\right)^{2}+2 A_{1}^{L} A_{2}^{L} \\
& -2 a . c A_{1}^{L}-2 a . c A_{2}^{L} \\
& +\left(A_{1}^{R}\right)^{2}+\left(A_{2}^{R}\right)^{2}+2 A_{1}^{R} A_{2}^{R}+(\text { a.c })^{2} \\
& -2 a . c A_{1}^{R}-2 a . c A_{2}^{R} \\
& =(a . c)^{2}+\left(A_{1}^{L}\right)^{2}-2 a \cdot c A_{1}^{L}+\left(A_{1}^{R}\right)^{2}+(a . c)^{2} \\
& -2 a . c A_{1}^{R}-(a . c)^{2}+(a . c)^{2}+\left(A_{2}^{L}\right)^{2} \\
& -2 a . c A_{2}^{L}-(a . c)^{2}+(a . c)^{2}+\left(A_{2}^{R}\right)^{2}-2 a . c A_{2}^{R} \\
& +2\left(A_{1}^{L} A_{2}^{L}+A_{1}^{R} A_{2}^{R}\right) \\
& =\left(a . c-A_{1}^{L}\right)^{2}+\left(A_{1}^{R}-a . c\right)^{2}+\left(a . c-A_{2}^{L}\right)^{2} \\
& +\left(A_{2}^{R}-a . c\right)^{2}-2\left[(a . c)^{2}+A_{1}^{L} A_{2}^{L}+A_{1}^{R} A_{2}^{R}\right] \\
& d^{2}\left(\alpha_{\left(A_{1}+A_{2}\right)}, \tilde{0}\right)<d^{2}\left(\alpha_{A_{1}}, \tilde{0}\right)+d^{2}\left(\alpha_{A_{2}}, \tilde{0}\right)
\end{aligned}
$$

Property 3.3. $d^{2}\left(k A_{1}, k A_{2}\right)=|k|^{2} d^{2}\left(A_{1}, A_{2}\right)$ for every $A_{1}, A_{2} \in$ $F$ and $k \in R$

Proof. $d^{2}\left(k A_{1}, k A_{2}\right)=\left(k a . c-k A_{1}^{L}\right)^{2}+\left(k A_{1}^{R}-k a . c\right)^{2}-[(k a . c-$ $\left.\left.k A_{2}^{L}\right)^{2}+\left(k A_{2}^{R}-k a . c\right)^{2}\right]$

$=(k a \cdot c)^{2}+\left(k A_{1}^{L}\right)^{2}-2 k^{2} a \cdot c \cdot A_{1}^{L}+\left(k A_{1}^{R}\right)^{2}+(k a \cdot c)^{2}-2 k^{2} a \cdot c A_{1}^{R}-$ $(k a . c)^{2}-\left(k A_{2}^{L}\right)^{2}+2 k^{2}$ a.c. $A_{2}^{L}-\left(k A_{2}^{R}\right)^{2}-(k a . c) 2^{+} 2 k^{2} a . c A_{2}^{R}$

$=k^{2}\left[(a . c)^{2}+A_{1}^{L^{2}}-2 a . c A_{1}^{L}+A_{1}^{R^{2}}+(\text { a.c })^{2}-2 a . c A_{1}^{R}-\left((a . c)^{2}+\right.\right.$ $\left.\left.A_{2}^{L^{2}}-2 a \cdot c A_{2}^{L}+A_{2}^{R^{2}}+(a \cdot c)^{2}-2 a \cdot c A_{2}^{R}\right)\right]$

$=k^{2}\left[\left(a . c-A_{1}^{L}\right)^{2}+\left(A_{1}^{R}-a . c\right)^{2}-\left(a . c-A_{2}^{L}\right)^{2}-\left(A_{2}^{R}-a . c\right)^{2}\right]$ $=|k|^{2} d^{2}\left(A_{1}, A_{2}\right)$

Here $\tilde{L}_{1}>\tilde{L}_{2}$ if and only if $A\left(\tilde{L}_{\max }>\tilde{L}_{1}\right)>A\left(\tilde{L}_{\max }>\tilde{L}_{2}\right)$

Since $b_{i}<x<c_{i}$,

$$
y_{d}=\frac{\left(c_{i}-x\right)}{\left(c_{i}-b_{i}\right)} \Rightarrow\left(c_{i}-b_{i}\right) y_{d}=c_{i}-x \Rightarrow x=c_{i}-\left(c_{i}-b_{i}\right) y_{d}
$$

Since $a<x<b$,

$$
y_{d}=\frac{(x-a)}{(b-a)} \Rightarrow(b-a) y_{d}=x-a \Rightarrow x=a+(b-a) y_{d}
$$

Equating (3.2) \& (3.3)

$c_{i}-\left(c_{i}-b_{i}\right) y_{d}=a+(b-a) y_{d}$

$y_{d}=\frac{\left(c_{i}-a\right)}{\left.(b-a)+\left(c_{i}-b_{(} i\right)\right)}$

\section{Application in critical path method}

\section{Notation 4.1.}

$N=$ The set of all nodes in a project network.

$A_{i j}=$ The Activity between nodes $i$ and $j$.

$F \tilde{E} T_{i j}=$ The fuzzy activity time of $A_{i j}$.

$F \tilde{E} S_{j}=$ The earliest starting fuzzy time of node $j$.

$F \tilde{L} F_{i}=$ The latest finishing fuzzy time of node $i$.

$F \tilde{T} S_{i j}=$ The total slack fuzzy time of $A_{i j}$.

\section{Definition 4.2. (Forward Pass Calculation)}

The earliest starting fuzzy time in the project network is calculated as follows:

Set the initial node to zero by starting (i.e)F $\tilde{E} S_{1}=(0,0,0)$ and

$F \tilde{E} S_{j}=\max _{i}\left\{F \tilde{E} S_{i}(+) F \tilde{E} T_{i j}\right\}, j \neq i . j \in N$

where $i=$ number of preceding nodes.

Ranking value is utilized to identify the maximum value.

Earliest finishing fuzzy time $=$ Earliest starting fuzzy time (+) fuzzy activity time.

Definition 4.3. (Backward Pass Calculation)

To calculate the latest finishing time in the project network.

Set $F \tilde{L} F_{n}=F \tilde{E} S_{n}$ and $F \tilde{L} F_{i}=\min _{j}\left\{F \tilde{L} F_{j}(-) F \tilde{E} T_{i j}\right\}, i \neq$ $n, i \in N$ and

where $j=$ number of succeeding nodes.

\subsection{New algorithm to find critical path}


1. Step1: Construct an acyclic network G(V,E), where V is the set of vertices and $\mathrm{E}$ is the set of edges. Let thestarting node always be $(0,0,0)$ denoted by a triangular fuzzy number.

2. Step2: Calculate the $E S_{i}$ for each node $i=1,2, \ldots, n$

3. Step3: Calculate the $\alpha$-cut for each $E S_{i}$ of each node.

4. Step4: Calculate the distance between the two nodes using Definition(3.1) which yields the duration for each activity which is a crisp number.

5. Step5: The fuzzy network converted to a crisp network

6. Step6: Compute $E S_{i}, L F_{j}$ and total float.

7. Step7: The activity with zero total float is the critical activity.

8. Step8: The path constituting critical activities form the critical path.

\subsection{Numerical Example}

Consider the example

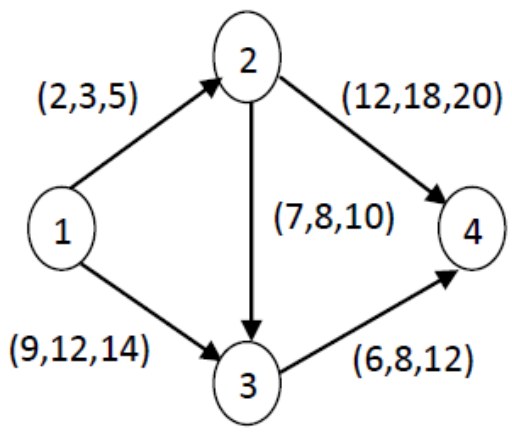

The corresponding fuzzy duration times are calculated using distance Definition(3.1) as follows

$$
\begin{aligned}
D^{2}\left(A_{1}, A_{2}\right) & =\int_{0}^{1 / 2}(1-\alpha)\left[\left(a . c-A_{1}^{L}(\alpha)\right)^{2}\right. \\
& \left.+\left(A_{1}^{R}(\alpha)-a . c\right)^{2}-\left[\left(a . c-A_{2}^{L}(\alpha)\right)^{2}+\left(A_{2}^{R}(\alpha)-a . c\right)^{2}\right]\right] d \alpha \\
& +\int_{1 / 2}^{1} \alpha\left[\left(a . c-A_{1}^{L}(\alpha)\right)^{2}+\left(A_{1}^{R}(\alpha)-a . c\right)^{2}\right. \\
& \left.-\left[\left(a . c-A_{2}^{L}(\alpha)\right)^{2}+\left(A_{2}^{R}(\alpha)-a . c\right)^{2}\right]\right] d \alpha \\
& =\int_{0}^{1 / 2}(1-\alpha)\left[-\left(\frac{7-\alpha}{2}-a \alpha-2\right)^{2}-\left(5-2 \alpha-\frac{7}{2}+\frac{\alpha}{2}\right)^{2}\right] d \alpha \\
& +\int_{1 / 2}^{1} \alpha\left[-\left(\frac{7-\alpha}{2}-a \alpha-2\right)^{2}-\left(5-2 \alpha-\frac{7}{2}+\frac{\alpha}{2}\right)^{2}\right] d \alpha \\
D_{2}\left(A_{1}, A_{2}\right)=-1.1719 & \\
D_{2}\left(A_{2}, A_{3}\right) & =\int_{0}^{1 / 2}(1-\alpha)\left[\left(\frac{-\alpha+7}{2}-(\alpha+2)\right)^{2}+\left(5-2 \alpha-\left(\frac{-\alpha+7}{2}\right)\right)^{2}\right. \\
& \left.-\left(\frac{3 \alpha-39}{2}-(7 \alpha+4)\right)^{2}-\left(25-4 \alpha-\left(\frac{3 \alpha+39}{2}\right)\right)^{2}\right] d \alpha \\
& +\int_{1 / 2}^{1} \alpha\left[\left(\frac{-\alpha+7}{2}-(\alpha+2)\right)^{2}+\left(5-2 \alpha-\left(\frac{-\alpha+7}{2}\right)\right)^{2}\right. \\
& -\left(\frac{3 \alpha+39}{2}-(7 \alpha+14)\right)^{2}-\left(25-4 \alpha-\left(\frac{3 \alpha+39}{2}\right)\right]^{2} d \alpha \\
& =-14.583
\end{aligned}
$$

Similarly,

$D_{2}\left(A_{2}, A_{4}\right)=-2.0837$

$D_{2}\left(A_{3}, A_{4}\right)=12.5$

$D_{2}\left(A_{1}, A_{4}\right)=3.255$

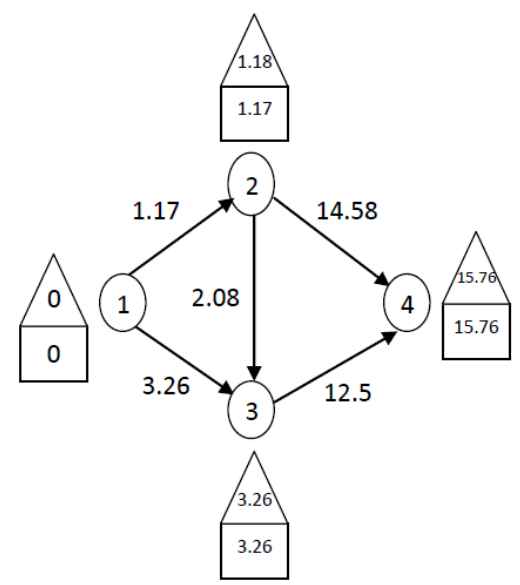

The Critical path is calculated as follows

\begin{tabular}{|l|l|l|l|l|l|l|l|}
\hline \multirow{2}{*}{ Activity } & \multirow{2}{*}{$\begin{array}{c}\text { Normal } \\
\text { time }\end{array}$} & \multicolumn{2}{|c|}{ Earliest } & \multicolumn{2}{|c|}{ Latest } & \multirow{2}{*}{ TF } & \multirow{2}{*}{ FF } \\
\cline { 3 - 7 } & Stant & Fimish & Stait & Finish & & \\
\hline $1-2$ & 1.17 & 0 & 1.17 & 0.01 & 1.18 & 0.02 & 0 \\
\hline $1-3$ & 3.26 & 0 & 3.26 & 0 & 3.26 & 0 & 0 \\
\hline $2-4$ & 14.58 & 1.17 & 15.75 & 1.18 & 15.76 & 0.01 & 0 \\
\hline 2.3 & 2.08 & 1.17 & 3.25 & 1.18 & 3.26 & 0.01 & 0 \\
\hline $3-4$ & 12.5 & 3.26 & 15.76 & 3.26 & 15.76 & 0 & 0 \\
\hline
\end{tabular}

The critical path is 1-3-4.

\section{References}

[1] Abbasbandy, S., Hajjari, T.: A new approach for ranking of trapezoidal fuzzy numbers, Comput. Math. Appl., 57(2009), 413-419.

[2] Chakraborty, C., Chakraborty, D.: A theoretical development on a fuzzy distance measure for fuzzy numbers, Math. Comput. Modeling, 43(2006), 254-261.

[3] Cheng, C.H.: A new approach for ranking fuzzy numbers by distance method, Fuzzy Sets and Systems, 95(1998), 307-317.

[4] Grzegorzewski, P.: Distances between intuitionistic fuzzy sets and/or interval-valued fuzzy sets based on the Hausdorff metric, Fuzzy Sets and Systems, 148(2004), 319328.

[5] Tran, L., Duckstein, L.: Comparison of fuzzy numbers using a fuzzy distance measure, Fuzzy Sets and Systems, 130(2002), 331-341. 
[6] Voxman, W.: Some remarks on distances between fuzzy numbers, Fuzzy Sets and Systems, 100(1998), 353-365.

$\operatorname{ISSN}(\mathrm{P}): 2319-3786$

Malaya Journal of Matematik

ISSN(O):2321-5666

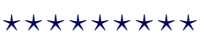

